BULLETIN (New Series) OF THE

AMERICAN MATHEMATICAL SOCIETY

Volume 36, Number 2, Pages 113-133

S 0273-0979(99)00775-2

Article electronically published on February 16, 1999

\title{
WHY THE CHARACTERISTIC POLYNOMIAL FACTORS
}

\author{
BRUCE E. SAGAN
}

\begin{abstract}
We survey three methods for proving that the characteristic polynomial of a finite ranked lattice factors over the nonnegative integers and indicate how they have evolved recently. The first technique uses geometric ideas and is based on Zaslavsky's theory of signed graphs. The second approach is algebraic and employs results of Saito and Terao about free hyperplane arrangements. Finally we consider a purely combinatorial theorem of Stanley about supersolvable lattices and its generalizations.
\end{abstract}

\section{INTRODUCTION}

The fundamental problem in enumerative combinatorics can be stated: given a set $S$, find a formula for its cardinality $|S|$. More generally, given a sequence of sets $S_{0}, S_{1}, S_{2}, \ldots$ we would like to investigate properties of the sequence

$$
a_{0}, a_{1}, a_{2}, \ldots
$$

where $a_{i}=\left|S_{i}\right|, i \geq 0$. From their definition we obviously have $a_{i} \in \mathbb{Z}_{\geq 0}$, the non-negative integers.

We can also turn this problem around. Suppose we are given a sequence (1) where the $a_{i}$ are defined in a way that would permit them to be in the complex numbers, $\mathbb{C}$. If, in fact, the terms are in $\mathbb{Z}_{>0}$, can we find a combinatorial explanation for this? One possibility, of course, would be to find a sequence of sets such that $a_{i}=\left|S_{i}\right|$. Such questions are of great interest currently in algebraic combinatorics.

We are going to investigate a particular problem of this type: trying to explain why the roots of a certain polynomial associated with a partially ordered set, called the characteristic polynomial, often has all of its roots in $\mathbb{Z}_{\geq 0}$. We will provide three explanations with tools drawn from three different areas of mathematics: graph theory/geometry, algebra, and pure combinatorics. The first of these uses Zaslavsky's lovely theory of signed graph coloring [57], [58], [59] which can be generalized to counting points of $\mathbb{Z}^{n}$ or of $\mathbb{F}_{p}^{n}$ inside a certain polytope [2], [15], [20], [26], [53]. (Here, $\mathbb{F}_{p}$ is the Galois field with $p$ elements.) The next technique is based on theorems of Saito [41] and Terao [51] about free hyperplane arrangements. Work has also been done on related concepts such as inductive freeness [50] and recursive freeness [60]. The third method employs a theorem of Stanley [44] on

Received by the editors June 30, 1996, and in revised form November 30, 1998.

1991 Mathematics Subject Classification. Primary 06A07; Secondary 05C15, 20F55, 06C10, 52 B30.

Key words and phrases. Characteristic polynomial, free arrangement, Möbius function, partially ordered set, signed graph, subspace arrangement, supersolvable lattice.

Presented at the 35th meeting of the Séminaire Lotharingien de Combinatoire, October 4-6, 1995.

(C)1999 American Mathematical Society 
semimodular supersolvable lattices which has recently been generalized by Blass and me [14] by relaxing both restrictions on the lattice. Along the way we will meet many important combinatorial concepts.

The rest of this paper is organized as follows. The next section will introduce the Möbius function, $\mu$, of a partially ordered set (poset) which is a far-reaching generalization of the one in number theory. In section 3 we will talk about generating functions, an important way to manipulate sequences such as (1), and define the characteristic polynomial, $\chi$, as the generating function for $\mu$. The last three sections will be devoted to the three methods for proving that, for various posets, $\chi$ factors over $\mathbb{Z}_{\geq 0}$.

\section{MöBius FUnCtions AND POSETS}

In number theory, one usually sees the Möbius function $\mu: \mathbb{Z}_{>0} \rightarrow \mathbb{Z}$ defined as

$$
\mu(n)= \begin{cases}0 & \text { if } n \text { is not square free, } \\ (-1)^{k} & \text { if } n \text { is the product of } k \text { distinct primes, }\end{cases}
$$

a definition which seems very strange at first blush. The importance of $\mu$ lies in the number-theoretic Möbius Inversion Theorem.

Theorem 2.1. Let $f, g: \mathbb{Z}_{>0} \rightarrow \mathbb{Z}$ satisfy

$$
f(n)=\sum_{d \mid n} g(d)
$$

for all $n \in \mathbb{Z}_{>0}$. Then

$$
g(n)=\sum_{d \mid n} \mu(n / d) f(d)
$$

Moving into the area of enumerative combinatorics, one of the very useful tools is the Principle of Inclusion-Exclusion or PIE.

Theorem 2.2. Let $S$ be a finite set and $S_{1}, \ldots, S_{n} \subseteq S$; then

$$
\left|S-\bigcup_{i=1}^{n} S_{i}\right|=|S|-\sum_{1 \leq i \leq n}\left|S_{i}\right|+\sum_{1 \leq i<j \leq n}\left|S_{i} \cap S_{j}\right|-\cdots+(-1)^{n}\left|\bigcap_{i=1}^{n} S_{i}\right| .
$$

In the theory of difference equations if one takes a function $f: \mathbb{Z}_{\geq 0} \rightarrow \mathbb{C}$, then there is an analog of the derivative, namely the difference operator

$$
\Delta f(n)=f(n)-f(n-1)
$$

(where $f(-1)=0$ by definition), and an analog of the integral, namely the summation operator

$$
S f(n)=\sum_{i=0}^{n} f(i) .
$$

The Fundamental Theorem of the Difference Calculus then states

Theorem 2.3. If $f: \mathbb{Z}_{\geq 0} \rightarrow \mathbb{C}$ then

$$
\Delta S f(n)=f(n) .
$$


$\{1,2,3\}$

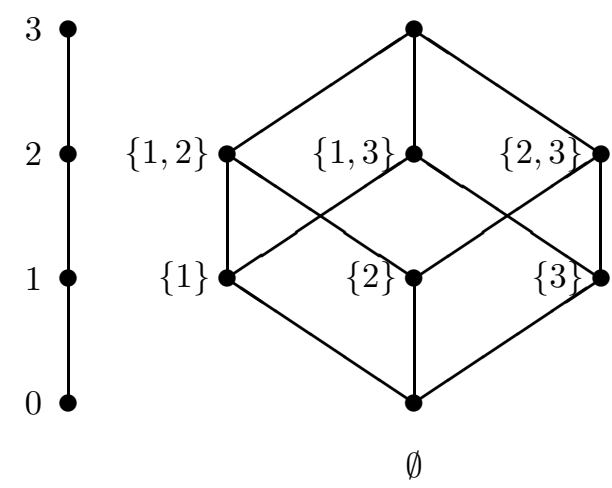

The chain $C_{3} \quad$ The Boolean algebra $B_{3}$

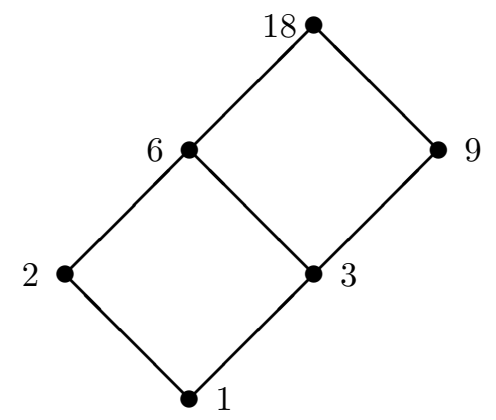

The divisor poset $D_{18}$

FIgURE 1. Some example posets

One of the advantages of the combinatorial Möbius function is that its inversion theorem unifies and generalizes the previous three results. In addition, it makes the definition (2) transparent, encodes topological information about posets [6], [40], and has even been used to bound the running time of certain algorithms [9]. We will now define this powerful invariant.

Let $P$ be a finite poset with partial order $\leq$. If $P$ has a unique minimal element, then it will be denoted $\hat{0}=\hat{0}_{P}$; and if it has a unique maximal element, then we will use the notation $\hat{1}=\hat{1}_{P}$. If $x \leq y$ in $P$, then the corresponding (closed) interval is

$$
[x, y]=\{z: x \leq z \leq y\}
$$

and we let $\operatorname{Int}(P)$ denote the set of all intervals of $P$. Note that $[x, y]$ is a poset in its own right with $\hat{0}_{[x, y]}=x, \hat{1}_{[x, y]}=y$. The Möbius function of $P, \mu: \operatorname{Int}(P) \rightarrow \mathbf{Z}$, is defined recursively by

$$
\mu(x, y)= \begin{cases}1 & \text { if } x=y \\ -\sum_{x \leq z<y} \mu(x, z) & \text { if } x<y\end{cases}
$$

Equivalently

$$
\sum_{x \leq z \leq y} \mu(x, z)=\delta_{x, y}
$$

where $\delta_{x, y}$ is the Kronecker delta. If $P$ has a zero, then we define $\mu(x)=\mu(\hat{0}, x)$.

Let us compute $\mu(x)$ in some simple posets. The chain, $C_{n}$, consists of the integers $\{0,1, \ldots, n\}$ ordered in the usual manner; see Figure 1 for a picture of $C_{3}$. It is immediate directly from the definition (3) that in $C_{n}$ we have

$$
\mu(x)= \begin{cases}1 & \text { if } x=0 \\ -1 & \text { if } x=1 \\ 0 & \text { if } x \geq 2\end{cases}
$$

The Boolean algebra, $B_{n}$, has as elements all subsets of $[n]:=\{1,2, \ldots, n\}$ and $\subseteq$ as order relation, the case $n=3$ being displayed in Figure 1. After computing the Möbius function of $B_{3}$, the reader will immediately guess that if $x \in B_{n}$ then $\mu(x)=(-1)^{|x|}$. This follows easily from the following observations. The Cartesian 
product of two posets $P, Q$ is obtained by ordering the $(x, y) \in P \times Q$ componentwise. It is easy to prove directly from (3) that if $\hat{0}_{P}$ and $\hat{0}_{Q}$ exist, then

$$
\mu_{P \times Q}(x, y)=\mu_{P}(x) \mu_{Q}(y) .
$$

Since $B_{n}$ is isomorphic as a poset to the $n$-fold product $\left(C_{1}\right)^{n}$, it is a simple matter to verify that its Möbius function has the desired form using equation (5).

The divisor poset, $D_{n}$, consists of all $d \mid n$ ordered by $c \leq d$ if $c \mid d$. Figure 1 shows $D_{18}$. Clearly if $n$ has prime factorization $n=\prod_{i} p_{i}^{n_{i}}$, then we have an isomorphism $D_{n} \cong \times_{i} C_{n_{i}}$. So as with the Boolean algebra, we get the value of $\mu(d)$ to be as in definition (2), this time in a much more natural way.

In case the reader is not convinced that the definition (4) is natural, consider the incidence algebra of $P, I(P)$, which consists of all functions $f: \operatorname{Int}(P) \rightarrow \mathbb{C}$. The multiplication in this algebra is convolution defined by

$$
f * g(x, y)=\sum_{x \leq z \leq y} f(x, z) g(z, y) .
$$

Note that with this multiplication $I(P)$ has an identity element $\delta: \operatorname{Int}(P) \rightarrow \mathbb{C}$, namely $\delta(x, y)=\delta_{x, y}$. One of the simplest but most important functions in $I(P)$ is the zeta function (so called because in $I\left(D_{n}\right)$ it is related to the Riemann zeta function) given by $\zeta(x, y)=1$ for all intervals $[x, y]$. It is easy to see that $\zeta$ is invertible in $I(P)$ and in fact that $\zeta^{-1}=\mu$ where $\mu$ is defined by (4).

The fundamental result about $\mu$ is the combinatorial Möbius Inversion Theorem [40].

Theorem 2.4. Let $P$ be a finite poset and $f, g: P \rightarrow \mathbb{C}$.

1. If for all $x \in P$ we have $f(x)=\sum_{y \leq x} g(y)$, then

$$
g(x)=\sum_{y \leq x} \mu(y, x) f(y) .
$$

2. If for all $x \in P$ we have $f(x)=\sum_{y \geq x} g(y)$, then

$$
g(x)=\sum_{y \geq x} \mu(x, y) f(y) .
$$

It is now easy to obtain the Theorems $2.1,2.2$, and 2.3 as corollaries by using Möbius inversion over $D_{n}, B_{n}$, and $C_{n}$, respectively. For example, to get the Principle of Inclusion-Exclusion, use $f, g: B_{n} \rightarrow \mathbb{Z}_{\geq 0}$ defined by

$$
\begin{aligned}
& f(X)=\left|S_{X}\right|, \\
& g(X)=\left|S_{X}-\bigcup_{i \notin X} S_{i}\right|,
\end{aligned}
$$

where $S_{X}=\cap_{i \in X} S_{i}$.

\section{Generating Functions And Characteristic polynomials}

The (ordinary) generating function for the sequence (1) is the formal power series

$$
f(x)=a_{0}+a_{1} x+a_{2} x^{2}+\cdots .
$$

Generating functions are a powerful tool for studying sequences, and Wilf has written a wonderful text devoted entirely to their study [55]. There are several reasons why one might wish to convert a sequence into its generating function. It may be possible to find a closed form for $f(x)$ when one does not exist for $\left(a_{n}\right)_{n \geq 0}$, 
or the expression for the generating function may be used to derive one for the sequence. Also, sometimes it is easier to obtain various properties of the $a_{n}$, such as a recursion or congruence relation, from $f(x)$ rather than directly.

By way of illustration, consider the sequence whose terms are

$$
p(n)=\text { number of integer partitions of the number } n \in \mathbb{Z}_{\geq 0}
$$

where an integer partition is a way of writing $n$ as an unordered sum of positive integers. For example, $p(4)=5$ because of the partitions

$$
4,3+1,2+2,2+1+1,1+1+1+1+1 .
$$

There is no known closed form for $p(n)$, but the generating function was found by Euler:

$$
\sum_{n=0}^{\infty} p(n) x^{n}=\prod_{k=1}^{\infty} \frac{1}{1-x^{k}}
$$

To see this, note that $1 /\left(1-x^{k}\right)=1+x^{k}+x^{2 k}+\cdots$, and so a term in the product obtained by choosing $x^{i k}$ from this expansion corresponds to choosing a partition with the integer $k$ repeated $i$ times. From (6) one can obtain all sorts of information about $p(n)$, such as its asymptotic behavior. See Andrews' book [1] for more details.

Our main object of study will be the generating function for the Möbius function of a poset, $P$, the so-called characteristic polynomial. Let $P$ have a zero and be ranked so that for any $x \in P$ all maximal chains from $\hat{0}$ to $x$ have the same length denoted $\rho(x)$ and called the rank of $x$. (A chain is a totally ordered subset of $P$, and maximal refers to inclusion.) The characteristic polynomial of $P$ is then

$$
\chi(P, t)=\sum_{x \in P} \mu(x) t^{\rho(\hat{1})-\rho(x)} .
$$

Note that we use the corank rather than the rank in the exponent on $t$ so that $\chi$ will be monic.

Let us look at some examples of posets and their characteristic polynomials, starting with those from the previous section. For the chain we clearly have

$$
\chi\left(C_{n}, t\right)=t^{n}-t^{n-1}=t^{n-1}(t-1) .
$$

Now for the Boolean algebra we have

$$
\chi\left(B_{n}, t\right)=\sum_{x \subseteq[n]}(-1)^{|x|} t^{n-|x|}=(t-1)^{n} .
$$

Note that by the same argument, if $k$ is the number of distinct primes dividing $n$, then

$$
\chi\left(D_{n}, t\right)=t^{\rho(n)-k}(t-1)^{k}
$$

since the terms divisible by squares contribute nothing to the sum. As a fourth example, consider the partition poset, $\Pi_{n}$, which consists of all set partitions of $[n]$ (families of disjoint nonempty subsets whose union is $[n]$ ) ordered by refinement. Direct computation with $\Pi_{3}$ (see Figure 2) shows that $\chi\left(\Pi_{3}, t\right)=t^{2}-3 t+2=$ $(t-1)(t-2)$. In general

$$
\chi\left(\Pi_{n}, t\right)=(t-1)(t-2) \cdots(t-n+1) .
$$

Note that in all cases $\chi$ has only nonnegative integral roots. 


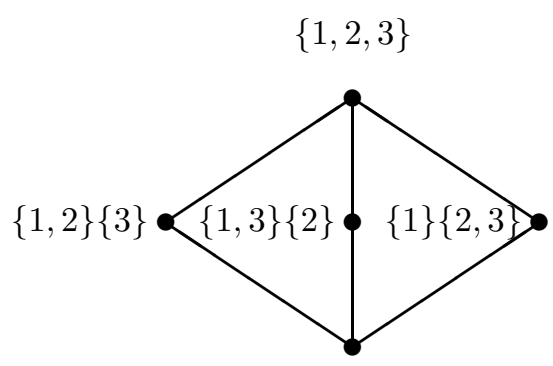

$\{1\}\{2\}\{3\}$

Figure 2. The partition poset $\Pi_{3}$

Many of our example posets will arise as intersection lattices of subspace arrangements. A lattice, $L$, is poset such that every pair $x, y \in L$ has a meet or greatest lower bound, $x \wedge y$, and a join or least upper bound, $x \vee y$. All our lattices will be finite and so will automatically have a zero $\hat{0}=\bigwedge L$ and a one $\hat{1}=\bigvee L$. A subspace arrangement is a finite set

$$
\mathcal{A}=\left\{K_{1}, K_{2}, \ldots, K_{l}\right\}
$$

of subspaces of real Euclidean space $\mathbb{R}^{n}$. If $\operatorname{dim} K_{i}=n-1$ for $1 \leq i \leq l$, then we say that $\mathcal{A}$ is a hyperplane arrangement and use $H$ 's in place of $K$ 's. The intersection lattice of $\mathcal{A}, L(\mathcal{A})$, has as elements all subspaces $X \subseteq \mathbb{R}^{n}$ that can be written as an intersection of some of the elements of $\mathcal{A}$. The partial order is reverse inclusion, so that $X \leq Y$ if and only if $X \supseteq Y$. So $L(\mathcal{A})$ has minimal element $\mathbb{R}^{n}$, maximal element $K_{1} \cap \cdots \cap K_{l}$, and join operation $X \vee Y=X \cap Y$. The reader can consult [7], [36] for more details about the general theory of arrangements which is currently a very active field.

The characteristic polynomial of $A$ is defined by

$$
\chi(\mathcal{A}, t)=\sum_{X \in L(\mathcal{A})} \mu(X) t^{\operatorname{dim} X} .
$$

This is not necessarily the same as $\chi(L(\mathcal{A}), t)$ as defined in (7). If $\mathcal{A}$ is a hyperplane arrangement, then the two will be equal up to a factor of a power of $t$, so from the point of view of having integral roots there is no difference. In the general subspace case (7) and (9) may be quite dissimilar, and often the latter turns out to factor at least partially over $\mathbb{Z}_{>0}$ while the former does not. In the arrangement, case the roots of (9) are called the exponents of $\mathcal{A}$ and denoted $\exp \mathcal{A}$. In fact when $\mathcal{A}$ is the set of reflecting hyperplanes for a Weyl group $W$, then these roots are just the usual exponents of $W[51]$ which are always nonnegative integers. The reason that Weyl groups, as opposed to more general Coxeter groups, have well-behaved characteristic polynomials is that such groups stabilize an appropriate discrete subgroup of $\mathbb{Z}^{n}$.

All of our previous example lattices can be realized as intersection lattices of subspace arrangements. The $n$-chain is $L(\mathcal{A})$ with $\mathcal{A}=\left\{K_{0}, \ldots, K_{n}\right\}$ where $K_{i}$ is the set of all points having the first $i$ coordinates zero. The Boolean algebra is the intersection lattice of the arrangement of coordinate hyperplanes $H_{i}: x_{i}=0$, $1 \leq i \leq n$. By combining these two constructions, one can also obtain a realization of the divisor poset as a subspace arrangement. To get the partition lattice we use 


\begin{tabular}{l|l|l}
$\mathcal{A}$ & $\chi(\mathcal{A}, t)$ & $\exp \mathcal{A}$ \\
\hline $\mathcal{A}_{n}$ & $t(t-1)(t-2) \cdots(t-n+1)$ & $0,1,2, \ldots, n-1$ \\
$\mathcal{B}_{n}$ & $(t-1)(t-3) \cdots(t-2 n+1)$ & $1,3,5, \ldots, 2 n-1$ \\
$\mathcal{D}_{n}$ & $(t-1)(t-3) \cdots(t-2 n+3)(t-n+1)$ & $1,3,5, \ldots, 2 n-3, n-1$
\end{tabular}

TABLE 1. Characteristic polynomials and exponents of some Weyl arrangements

the Weyl arrangement of type $A$

$$
\mathcal{A}_{n}=\left\{x_{i}-x_{j}=0: 1 \leq i<j \leq n\right\} .
$$

To see why $\Pi_{n}$ and $L\left(\mathcal{A}_{n}\right)$ are the same, associate the hyperplane $x_{i}=x_{j}$ with the partition where $i, j$ are in one subset and all other subsets are singletons. This will then make the join operations in the two lattices correspond. Note that the characteristic polynomials defined by (7) and (9) are the same in the first two examples while $\chi\left(\mathcal{A}_{n}, t\right)=t \chi\left(\Pi_{n}, t\right)$.

We will also be concerned with the hyperplane arrangements associated with other Weyl groups. The reader interested in more information about these groups should consult the excellent text of Humphreys [30]. In particular, the other two infinite families

$$
\begin{aligned}
& \mathcal{B}_{n}=\left\{x_{i} \pm x_{j}=0: 1 \leq i<j \leq n\right\} \cup\left\{x_{i}=0: 1 \leq i \leq n\right\} \\
& \mathcal{D}_{n}=\left\{x_{i} \pm x_{j}=0: 1 \leq i<j \leq n\right\}
\end{aligned}
$$

will play a role. The corresponding characteristic polynomials are listed in Table 1 along with $\chi\left(\mathcal{A}_{n}, t\right)$ for completeness. (We do not consider the arrangement for the root system $C_{n}$ because its roots are scalar multiples of the ones for $B_{n}$, thus yielding the same arrangement.) We will show how to derive the formulas for the characteristic polynomials of $\mathcal{A}_{n}, \mathcal{B}_{n}$ and $\mathcal{D}_{n}$ using elementary graph theory in the next section.

\section{Signed GRAPHS}

Zaslavsky developed his theory of signed graphs [57], [58], [59] to study hyperplane arrangements contained in the Weyl arrangement $\mathcal{B}_{n}$. (Note that this includes $\mathcal{A}_{n}$ and $\left.\mathcal{D}_{n}.\right)$ In particular his coloring arguments provide one of the simplest ways to compute the corresponding characteristic polynomials.

A signed graph, $G=(V, E)$, consists of a set $V$ of vertices which we will always take to be $\{1,2, \ldots, n\}$, and a set of edges $E$ which can be of three possible types:

1. a positive edge between $i, j \in V$, denoted $i j^{+}$,

2. a negative edge between $i, j \in V$, denoted $i j^{-}$,

3. or a half-edge which has only one endpoint $i \in V$, denoted $i^{h}$.

One can have both the positive and negative edges between a given pair of vertices, in which case it is called a doubled edge and denoted $i j^{ \pm}$. The three types of edges correspond to the three types of hyperplanes in $\mathcal{B}_{n}$, namely $x_{i}=x_{j}, x_{i}=-x_{j}$, and $x_{i}=0$ for the positive, negative, and half-edges, respectively. So to every hyperplane arrangement $\mathcal{A} \subseteq \mathcal{B}_{n}$ we have an associated signed graph $G_{\mathcal{A}}$ with a hyperplane in $\mathcal{A}$ if and only if the corresponding edge is in $G_{\mathcal{A}}$. Actually, the possible edges in $G_{\mathcal{A}}$ really correspond to the vectors in the root system of type $B_{n}$ perpendicular to the hyperplanes, which are $e_{i}-e_{j}, e_{i}+e_{j}$, and $e_{i}$. (In the full theory one also considers the root system $C_{n}$ with roots $2 e_{i}$ which are modeled 


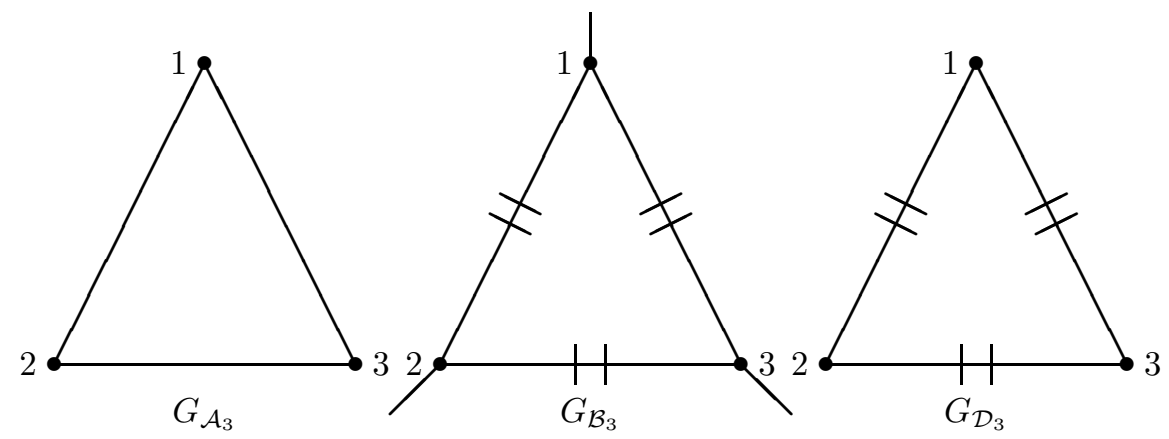

FiguRE 3. Graphs for Weyl arrangements

by loops $i i$ in $G$. This is why the somewhat strange definition of a half-edge is necessary. Loops and half-edges behave differently because, e.g., the former can be in a circuit of the graph while the later cannot.) In picturing a signed graph I will draw an ordinary edge for $i j^{+}$, an edge with a slash through it for $i j^{-}$, an edge with two slashes through it for $i j^{ \pm}$, and an edge starting at a vertex and wandering off into space for $i^{h}$. The graphs $G_{\mathcal{A}_{3}}, G_{\mathcal{B}_{3}}$, and $G_{\mathcal{D}_{3}}$ are shown in Figure 3.

Since we are using signed edges, we are also going to use signed colors for the vertices. For $s \in \mathbb{Z}_{\geq 0}$ let $[-s, s]=\{-s,-s+1, \ldots, s-1, s\}$. A coloring of the signed graph $G$ is a function $c: V \rightarrow[-s, s]$. The fact that the number of colors $t=|[-s, s]|=2 s+1$ is always odd will be of significance later. A proper coloring $c$ of $G$ requires that for every edge $e \in E$ we have

1. if $e=i j^{+}$, then $c(i) \neq c(j)$;

2. if $e=i j^{-}$, then $c(i) \neq-c(j)$;

3. if $e=i^{h}$, then $c(i) \neq 0$.

Note that the first of these three restrictions is the one associated with ordinary graphs and the four-color theorem [19]. The chromatic polynomial of $G$ is

$$
P(G, t)=\text { the number of proper colorings of } G \text { with } t \text { colors. }
$$

It is not obvious from the definition that $P(G, t)$ is actually a polynomial in $t$. In fact even more is true as we see in the following theorem of Zaslavsky.

Theorem 4.1 ([58]). Suppose $\mathcal{A} \subseteq \mathcal{B}_{n}$ has signed graph $G_{\mathcal{A}}$. Then

$$
\chi(\mathcal{A}, t)=P\left(G_{\mathcal{A}}, t\right) .
$$

Theorem 4.1 trivializes the calculation of the characteristic polynomials for the three infinite families of Weyl arrangements and in so doing explains why they factor over $\mathbb{Z}_{\geq 0}$. For $\mathcal{A}_{n}$ the graph $G_{\mathcal{A}_{n}}$ consists of every possible positive edge. So to properly color $G_{\mathcal{A}_{n}}$ we have $t$ choices for vertex 1 ; then $t-1$ for vertex 2 since $c(2) \neq c(1)$, and so forth, yielding

$$
\chi\left(\mathcal{A}_{n}, t\right)=P\left(G_{\mathcal{A}_{n}}\right)=t(t-1) \cdots(t-n+1)
$$

in agreement with Table 1 . It will be convenient in a bit to have a shorthand for this falling factorial, so let $\langle t\rangle_{n}=t(t-1) \cdots(t-n+1)$. In $G_{\mathcal{B}_{n}}$ we also have every negative edge and half-edge. This gives $t-1$ choices for vertex 1 since color 0 is not allowed; $t-3$ choices for vertex 2 since $c(2) \neq \pm c(1), 0$; and so on. These are exactly the factors in the $\mathcal{B}_{n}$ entry of Table 1 . Finally consider $G_{\mathcal{D}_{n}}$ which is 
just $G_{\mathcal{B}_{n}}$ with the half-edges removed. There are two cases depending on whether the color 0 appears once or not at all. (It can't appear two or more times because $G_{\mathcal{A}_{n}} \subseteq G_{\mathcal{D}_{n}}$.) If the color 0 is never used, then we have the same number of colorings as with $\mathcal{B}_{n}$. If 0 is used once, then there are $n$ vertices that could receive it and the rest are colored as in $\mathcal{B}_{n-1}$. So

$$
\chi\left(\mathcal{D}_{n}, t\right)=\prod_{i=1}^{n}(t-2 i+1)+n \prod_{i=1}^{n-1}(t-2 i+1)=(t-n+1) \prod_{i=1}^{n-1}(t-2 i+1),
$$

which again agrees with the table.

Blass and I have generalized Zaslavsky's theorem from hyperplane arrangements to subspace arrangements. If $\mathcal{A}$ and $\mathcal{B}$ are subspace arrangements, then we say that $\mathcal{A}$ is embedded in $\mathcal{B}$ if all subspaces of $\mathcal{A}$ are intersections of subspaces of $\mathcal{B}$, i.e., $\mathcal{A} \subseteq L(\mathcal{B})$. Now consider $[-s, s]^{n}$ as a cube of integer lattice points in $\mathcal{R}^{n}$ (not to be confused with our use of lattice as a type of partially ordered set). Let $[-s, s]^{n} \backslash \bigcup \mathcal{A}$ denote the set of points of the cube which lie on none of the subspaces in $\mathcal{A}$. We will include a proof of the next result because it amply illustrates the importance of the Möbius Inversion Theorem.

Theorem 4.2 ([15]). Let $t=2 s+1$ where $s \in \mathbb{Z}_{\geq 0}$ and let $\mathcal{A}$ be any subspace arrangement embedded in $\mathcal{B}_{n}$. Then

$$
\chi(\mathcal{A}, t)=\left|[-s, s]^{n} \backslash \bigcup \mathcal{A}\right| .
$$

Proof. We construct two functions $f, g: L(\mathcal{A}) \longrightarrow \mathbf{Z}$ by defining for each $X \in L(\mathcal{A})$

$$
\begin{aligned}
& f(X)=\left|X \cap[-s, s]^{n}\right|, \\
& g(X)=\left|\left(X \backslash \bigcup_{Y>X} Y\right) \cap[-s, s]^{n}\right| .
\end{aligned}
$$

Recall that $L(\mathcal{A})$ is ordered by reverse inclusion so that $\bigcup_{Y>X} Y \subset X$. In particular $g\left(\mathbb{R}^{n}\right)=\left|[-s, s]^{n} \backslash \bigcup \mathcal{A}\right|$. Note also that $X \cap[-s, s]^{n}$ is combinatorially just a cube of dimension $\operatorname{dim} X$ and side $t$ so that $f(X)=t^{\operatorname{dim} X}$. Finally, directly from our definitions, $f(X)=\sum_{Y \geq X} g(Y)$, so by the Theorem 2.4

$$
\begin{aligned}
\left|[-s, s]^{n} \backslash \bigcup \mathcal{A}\right| & =g(\hat{0}) \\
& =\sum_{X \geq \hat{0}} \mu(X) f(X) \\
& =\sum_{X \in L(\mathcal{A})} \mu(X) t^{\operatorname{dim} X} \\
& =\chi(\mathcal{A}, t),
\end{aligned}
$$

which is the desired result.

To see why our theorem implies Zaslavsky's in the hyperplane case, note that a point $c \in[-s, s]^{n}$ is just a coloring $c: V \rightarrow[-s, s]$ where the $i$ th coordinate of the point is the color of the vertex $i$. With this viewpoint, a coloring is proper if and only if the corresponding point is not on any hyperplane of $\mathcal{A}$. For example, if $i j^{+} \in E$ then the coloring must have $c(i) \neq c(j)$ and so the point does not lie on the hyperplane $x_{i}=x_{j}$. 
As an application of Theorem 4.2, we consider a set of subspace arrangements that has been arousing a lot of interest lately. The $k$-equal arrangement of type $A$ is

$$
\mathcal{A}_{n, k}=\left\{x_{i_{1}}=x_{i_{2}}=\ldots=x_{i_{k}}: 1 \leq i_{1}<i_{2}<\ldots<i_{k} \leq n\right\} .
$$

The $\mathcal{A}_{n, k}$ arrangements were introduced in the work of Björner, Lovász and Yao [9], motivated, surprisingly enough, by its relevance to a certain problem in computational complexity. Its study has been continued by many people including Linusson, Sundaram, Wachs and Welker [8], [13], [11], [12], [34], [35], [48], [49]. Analogs of this subspace arrangement for types $B$ and $D$ have also been studied by Björner and me [10].

Now in general $\chi\left(\mathcal{A}_{n, k}\right)$ does not factor completely over $\mathbb{Z}_{\geq 0}$, but it does factor partially. In fact it is divisible by the characteristic polynomial $\chi\left(\mathcal{A}_{m}, t\right)=\langle t\rangle_{m}$ for a certain $m$. What's more, if one expands $\chi\left(\mathcal{A}_{n, k}\right)$ in the basis $\langle t\rangle_{n}, n \geq 0$, for the polynomial ring, then the coefficients are nonnegative integers with a simple combinatorial interpretation. In particular, let $S_{k}(n, j)$ denote the number of partitions of an $n$-element set into $j$ subsets each of which is of size at most $k$. Thus these are generalizations of the Stirling numbers of the second kind. We now have the expansion, first derived by Sundaram [47]

$$
\chi\left(\mathcal{A}_{n, k}, t\right)=\sum_{j} S_{k-1}(n, j)\langle t\rangle_{j} .
$$

To see why this is true, consider an arbitrary point $c \in[-s, s]^{n} \backslash \bigcup \mathcal{A}_{n, k}$. So $c$ can have at most $k-1$ of its coordinates equal. Consider the $c$ 's with exactly $j$ different coordinates. Then there are $S_{k-1}(n, j)$ ways to distribute the $j$ values among the $n$ coordinates with at most $k-1$ equal. Next we can choose which values to use in $\langle t\rangle_{j}$ ways. Summing over all $j$ gives the desired equation. From (10) we can immediately derive a divisibility relation. To state it, let $\lceil\cdot\rceil$ be the ceiling or round up function. Then

$$
\langle t\rangle_{\lceil n /(k-1)\rceil} \mid \chi\left(\mathcal{A}_{n, k}, t\right)
$$

since $S_{k-1}(n, j)=0$ if $j<\lceil n /(k-1)\rceil$. (Obviously $j$ sets of at most $k-1$ elements can partition a set of size at most $n=j(k-1)$.)

Thinking about things in terms of lattice points also permits a generalization of Zaslavsky's theorem in another direction, namely to all Weyl hyperplane arrangements (even the exceptional ones). Let $\Phi \subset \mathbb{R}^{n}$ be a root system for a finite Weyl group $W$, and let $\mathcal{W}$ be the set of hyperplanes perpendicular to the roots. Let $(\cdot, \cdot)$ denote the standard inner product on $\mathbb{R}^{n}$. The role of the cube in Theorem 4.2 will be played by

$$
P_{t}(\Phi)=\left\{x \in \mathbb{R}^{n}:(x, \alpha) \in \mathbb{Z}_{<t} \text { for all } \alpha \in \Phi\right\},
$$

which is a set of points in the coweight lattice of $\Phi$ closely associated with the Weyl chambers of the corresponding affine Weyl group.

Consider a fixed simple system

$$
\Delta=\left\{\sigma_{1}, \ldots, \sigma_{n}\right\}
$$

in $\Phi$. Since $\Delta$ is a basis for $\mathbf{R}^{n}$, any root $\alpha \in \Phi$ can be written as a linear combination

$$
\alpha=\sum_{i=1}^{n} s_{i}(\alpha) \sigma_{i}
$$


In fact the coefficients $s_{i}(\alpha)$ are always integers. Among all the roots, there is a highest one, $\tilde{\alpha}$, characterized by the fact that for all roots $\alpha$ and all $i \in[n]$, $s_{i}(\tilde{\alpha}) \geq s_{i}(\alpha)$. We will also need a weighting factor called the index of connection, $f$, which is the index of the lattice generated by the roots in the coweight lattice. The second generalization can now be stated.

Theorem 4.3 ([2], [15], [26]). Let $\Phi$ be a root system for a finite Weyl group with associated arrangement $\mathcal{W}$. Let $t$ be a positive integer relatively prime to $s_{i}(\tilde{\alpha})$ for all $i$. Then

$$
\chi(\mathcal{W}, t)=\frac{1}{f}\left|P_{t}(\Phi) \backslash \bigcup \mathcal{W}\right|
$$

Note how the condition in Theorem 4.2 that $t$ be odd has been replaced by a relative primeness restriction. This is typical when dealing with Ehrhart quasipolynomials [45, page 235ff.] which enumerate the number of points of a given lattice inside a polytope and its blowups. We have not been able to use (11) to explain the factorization of $\chi(\mathcal{W}, t)$ over $\mathbf{Z}_{\geq 0}$ as was done with Theorem 4.1 for the three infinite families. It would be interesting if this hole could be filled.

Athanasiadis [2] has given a very pretty proof of the previous theorem. His main tool is a reworking of a result of Crapo and Rota [20] which is similar in statement and proof to Theorem 4.2 but replaces $[-s, s]^{n}$ by $\mathbb{F}_{p}^{n}$ where $\mathbb{F}_{p}$ is the finite field with $p$ elements, $p$ prime. Terao [53] also independently discovered this theorem.

Theorem 4.4 ([2], [20], [53]). Let $\mathcal{A}$ be any subspace arrangement in $\mathbb{R}^{n}$ defined over the integers and hence over $\mathbb{F}_{p}$. Then for large enough primes $p$ we have

$$
\chi(\mathcal{A}, p)=\left|\mathbb{F}_{p}^{n} \backslash \bigcup \mathcal{A}\right| \text {. }
$$

Athanasiadis has also used the previous result to give very elegant derivations of characteristic polynomials for many arrangements which cannot be handled by Theorem 4.2. For the rest of this section only, we will enlarge the definition of a hyperplane arrangement to be any finite set of affine hyperplanes (not necessarily passing through the origin). An arrangement $\mathcal{B}$ is a deformation of arrangement $\mathcal{A}$ if every hyperplane of $\mathcal{B}$ is parallel to some hyperplane of $\mathcal{A}$. As an example consider the Shi arrangement of type $A, \mathcal{S}_{n}$, with hyperplanes

$$
x_{i}-x_{j}=0, x_{i}-x_{j}=1, \quad \text { where } 1 \leq i<j \leq n,
$$

which is a deformation of the corresponding Weyl arrangement. Such arrangements were introduced by Shi [42], [43] for studying affine Weyl groups. Headley [27], [28] first computed their characteristic polynomials in a way that was universal for all types but relied on a formula of Shi's that had a complicated proof. To illustrate the power of Theorem 4.4, we will reproduce the proof in [2] that

$$
\chi\left(\mathcal{S}_{n}, t\right)=t(t-n)^{n-1} .
$$

Consider any $\left(x_{1}, \ldots, x_{n}\right) \in \mathbb{F}_{p}^{n}$ as a placement of balls labeled $1, \ldots, n$ into a circular array of boxes, labeled clockwise as $0, \ldots, p-1$, where placement of ball $i$ in box $j$ means $x_{i}=j$. Then $\left(x_{1}, \ldots, x_{n}\right) \in \mathbb{F}_{p}^{n} \backslash \bigcup \mathcal{S}_{n}$ means that no two balls are in the same box and that if two balls are in adjacent boxes, then they must be in increasing order clockwise. All such placements can be derived as follows. Take $p-n$ unlabeled boxes and place them in a circle. Now put the balls $1, \ldots, n$ into the spaces between the boxes so that adjacent ones increase clockwise. Note that since the boxes are unlabeled, there is only one way to place ball 1 , but once that 
is done balls $2, \ldots, n$ can be placed in $(p-n)^{n-1}$ ways. Finally, put an unlabeled box around each ball, label all the boxes clockwise as $0, \ldots, p-1$ which can be done in $p$ ways, and we are done.

The connected components of $\mathbb{R}^{n} \backslash \mathcal{A}$ are called regions, and a region is bounded if it is contained in some sphere about the origin. If we let $r(\mathcal{A})$ and $b(\mathcal{A})$ denote the number of regions and bounded regions, respectively, of $\mathcal{A}$, then we can state the following striking result of Zaslavsky.

Theorem 4.5 ([56]). For any affine hyperplane arrangement

$$
r(\mathcal{A})=|\chi(\mathcal{A},-1)|=\sum_{X \in L(\mathcal{A})}|\mu(X)|
$$

and

$$
b(\mathcal{A})=|\chi(\mathcal{A}, 1)|=\left|\sum_{X \in L(\mathcal{A})} \mu(X)\right| .
$$

Using the characteristic polynomials in Table 1 we see that

$$
\begin{array}{ll}
b\left(\mathcal{A}_{n}\right)=|(-1)(-1-1)(-1-2) \cdots(-1-n+1)| & =n ! \\
b\left(\mathcal{B}_{n}\right)=|(-1-1)(-1-3) \cdots(-1-2 n+1)| & =2^{n} n ! \\
b\left(\mathcal{C}_{n}\right)=|(-1-1)(-1-3) \cdots(-1-2 n+3)(-1+n+1)| & =2^{n-1} n !
\end{array}
$$

which agrees with the well-known fact that the number of chambers of a Weyl arrangement is the same as the number of elements in the corresponding group. It was Shi's formula for the number of regions in his arrangements that Headly needed to derive the full characteristic polynomial. In particular, it follows from (12) that $r\left(\mathcal{S}_{n}\right)=(n+1)^{n-1}$, which is known to be the number of labeled trees on $n+1$ vertices, or the number of parking functions of length $n$. Many people [3], [28], [37], [38], [46] have used these combinatorial interpretations to come up with bijective proofs of this formula and related ones.

\section{Free arrangements}

In this section we consider a large class of hyperplane arrangements called free arrangements which were introduced by Terao [51]. The characteristic polynomial of such an arrangement factors over $\mathbb{Z}_{\geq 0}$ because its roots are related to the degrees of basis elements for a certain associated free module.

Our modules will be over the polynomial algebra $A=\mathbb{R}\left[x_{1}, \ldots, x_{n}\right]=\mathbb{R}[x]$ graded by total degree so $A=\oplus_{i \geq 0} A_{i}$. The module of derivations, $D$, consists of all $\mathbb{R}$-linear maps $\theta: A \rightarrow A$ satisfying

$$
\theta(f g)=f \theta(g)+g \theta(f)
$$

for any $f, g \in A$. This module can be graded by saying that $\theta$ has degree $d$, $\operatorname{deg} \theta=d$, if $\theta\left(A_{i}\right) \subseteq A_{i+d}$ for all $i \geq 0$. Also, $D$ is free with basis $\partial / \partial x_{1}, \ldots, \partial / \partial x_{n}$. It is simplest to display a derivation as a column vector with entries being its components with respect to this basis. So if $\theta=p_{1}(x) \partial / \partial x_{1}+\cdots+p_{n}(x) \partial / \partial x_{n}$, then we write

$$
\theta=\left[\begin{array}{c}
p_{1}(x) \\
\vdots \\
p_{n}(x)
\end{array}\right]=\left[\begin{array}{c}
\theta\left(x_{1}\right) \\
\vdots \\
\theta\left(x_{n}\right)
\end{array}\right]
$$


Two operators that we will find useful are

$$
\mathbf{X}^{d}=x_{1}^{d} \partial / \partial x_{1}+\cdots+x_{n}^{d} \partial / \partial x_{n}=\left[\begin{array}{c}
x_{1}^{d} \\
\vdots \\
x_{n}^{d}
\end{array}\right]
$$

and

$$
\hat{\mathbf{X}}=\hat{x}_{1} \partial / \partial x_{1}+\cdots+\hat{x}_{n} \partial / \partial x_{n}=\left[\begin{array}{c}
\hat{x}_{1} \\
\vdots \\
\hat{x}_{n}
\end{array}\right]
$$

where $\hat{x}_{i}=x_{1} x_{2} \cdots x_{n} / x_{i}$. Note that $\operatorname{deg} \mathbf{X}^{d}=d-1$ and $\operatorname{deg} \hat{\mathbf{X}}=n-2$.

To see the connection with hyperplane arrangements, notice that any hyperplane $H$ is defined by a linear equation $\alpha_{H}\left(x_{1}, \ldots, x_{n}\right)=0$. It is then useful to study the associated module of $\mathcal{A}$-derivations, which is defined by

$$
D(\mathcal{A})=\left\{\theta \in D: \alpha_{H} \mid \theta\left(\alpha_{H}\right) \text { for all } H \in \mathcal{A}\right\}
$$

where $p \mid q$ is division of polynomials in $A$. By way of illustration, $\mathbf{X}^{d} \in D\left(\mathcal{A}_{n}\right)$ for all $d \geq 0$ since $\mathbf{X}^{d}\left(x_{i}-x_{j}\right)=x_{i}^{d}-x_{j}^{d}$ which is divisible by $x_{i}-x_{j}$. Similarly $\mathbf{X}^{2 d+1} \in D\left(\mathcal{D}_{n}\right)$ because of what we just showed for $\mathcal{A}_{n}$ and the fact that we have $x_{i}+x_{j} \mid x_{i}^{2 d+1}+x_{j}^{2 d+1}$. The $\mathbf{X}^{2 d+1}$ are also in $D\left(\mathcal{B}_{n}\right)$ since $x_{i} \mid x_{i}^{2 d+1}$. By the same methods we get $\hat{\mathbf{X}} \in D\left(\mathcal{D}_{n}\right)$.

We say that the arrangement $\mathcal{A}$ is free if $D(\mathcal{A})$ is free as a module over $A$. Freeness is intimately connected with the factorization of $\chi$ as the next theorem shows.

Theorem $5.1([51])$. If $\mathcal{A}$ is free, then $D(\mathcal{A})$ has a homogeneous basis $\theta_{1}, \ldots, \theta_{n}$ and the degree set $\left\{d_{1}, \ldots, d_{n}\right\}=\left\{\operatorname{deg} \theta_{1}, \ldots, \operatorname{deg} \theta_{n}\right\}$ depends only on $\mathcal{A}$. Furthermore

$$
\chi(\mathcal{A}, t)=\left(t-d_{1}-1\right) \cdots\left(t-d_{n}-1\right) .
$$

We have a simple way to check whether a derivation is in $D(\mathcal{A})$ for a given arrangement $\mathcal{A}$. It would be nice to have an easy way to test whether $\mathcal{A}$ is free and if so find a basis. This is the Saito Criterion. Given derivations $\theta_{1}, \ldots, \theta_{n}$, consider the matrix whose columns are the corresponding column vectors

$$
\Theta=\left[\theta_{1}, \ldots, \theta_{n}\right]=\left[\theta_{j}\left(x_{i}\right)\right] .
$$

Also consider the homogeneous polynomial

$$
Q=Q(\mathcal{A})=\prod_{H \in \mathcal{A}} \alpha_{H}(x)
$$

which has the arrangement $\mathcal{A}$ as zero set and is called its defining form. For example

$$
\begin{aligned}
Q\left(\mathcal{A}_{n}\right) & =\prod_{1 \leq i<j \leq n}\left(x_{i}-x_{j}\right) \\
Q\left(\mathcal{B}_{n}\right) & =x_{1} x_{2} \cdots x_{n} \prod_{1 \leq i<j \leq n}\left(x_{i}^{2}-x_{j}^{2}\right) \\
Q\left(\mathcal{D}_{n}\right) & =\prod_{1 \leq i<j \leq n}\left(x_{i}^{2}-x_{j}^{2}\right) .
\end{aligned}
$$


Theorem $5.2([41],[52])$. Suppose $\theta_{1}, \ldots, \theta_{n} \in D(\mathcal{A})$ and that $Q$ is the defining form of $\mathcal{A}$. Then $\mathcal{A}$ is free with basis $\theta_{1}, \ldots, \theta_{n}$ if and only if

$$
\operatorname{det} \Theta=c Q
$$

for some $c \in \mathbb{R} \backslash 0$.

How could this be applied to the Weyl arrangements? Given what we know about elements in their derivation modules and the factorization of their characteristic polynomials, it is natural to guess that we might be able to prove freeness with the following matrices:

$$
\begin{aligned}
\Theta\left(\mathcal{A}_{n}\right) & =\left[\mathbf{X}^{0}, \mathbf{X}^{1}, \mathbf{X}^{2}, \ldots, \mathbf{X}^{n-1}\right] \\
\Theta\left(\mathcal{B}_{n}\right) & =\left[\mathbf{X}^{1}, \mathbf{X}^{3}, \mathbf{X}^{5}, \ldots, \mathbf{X}^{2 n-1}\right] \\
\Theta\left(\mathcal{D}_{n}\right) & =\left[\mathbf{X}^{1}, \mathbf{X}^{3}, \mathbf{X}^{5}, \ldots, \mathbf{X}^{2 n-3}, \hat{\mathbf{X}}\right] .
\end{aligned}
$$

Of course $\operatorname{det} \Theta\left(\mathcal{A}_{n}\right)= \pm \prod_{1 \leq i<j \leq n}\left(x_{i}-x_{j}\right)=Q\left(\mathcal{A}_{n}\right)$ is just Vandermonde's determinant. Similarly we get $\operatorname{det} \Theta\left(\mathcal{B}_{n}\right)= \pm x_{1} x_{2} \cdots x_{n} \prod_{1 \leq i<j \leq n}\left(x_{i}^{2}-x_{j}^{2}\right)$ by first factoring out $x_{i}$ from the $i$ th row, which results in a Vandermonde in squared variables. For $\mathcal{D}_{n}$ just factor out $x_{1} x_{2} \cdots x_{n}$ from the last column and then put these factors back in by multiplying row $i$ by $x_{i}$. The result is again a Vandermonde in squares. Now the roots of the corresponding characteristic polynomials can be read off these matrices in agreement with Table 1.

The reader may have noticed that the bases we have for $D\left(\mathcal{B}_{n}\right)$ and $D\left(\mathcal{D}_{n}\right)$ are the same except for the last derivation. This is reflected in the fact that $\exp \mathcal{B}_{n}$ and $\exp \mathcal{D}_{n}$ are the same except for the last root. Note that the difference between these roots is $n$, which is exactly the number of hyperplanes in $\mathcal{B}_{n}$ but not in $\mathcal{D}_{n}$. Wouldn't it be lovely if adding these hyperplanes one at a time to $\mathcal{D}_{n}$ would produce a sequence of arrangements all of whose exponents agreed with $\exp \left(\mathcal{D}_{n}\right)$ except the last one, which would increase by one each time a hyperplane is added until we reach $\exp \left(\mathcal{B}_{n}\right)$ ? This is in fact what happens. Define

$$
\mathcal{D} B_{n, k}=\mathcal{D}_{n} \cup\left\{x_{1}, x_{2}, \ldots, x_{k}\right\}
$$

so that $\mathcal{D} B_{n, 0}=\mathcal{D}_{n}$ and $\mathcal{D} B_{n, n}=\mathcal{B}_{n}$. Now the derivation $\theta_{k}=x_{1} x_{2} \cdots x_{k} \hat{\mathbf{X}}$ (scalar multiplication) is in $D\left(\mathcal{D} B_{n, k}\right)$ since $\hat{\mathbf{X}} \in D\left(\mathcal{D}_{n}\right)$ and $x_{i} \mid \theta_{k}\left(x_{i}\right)$ for $1 \leq i \leq$ $k$. Furthermore, if we let

$$
\Theta\left(\mathcal{D} B_{n, k}\right)=\left[\mathbf{X}^{1}, \mathbf{X}^{3}, \mathbf{X}^{5}, \ldots, \mathbf{X}^{2 n-3}, \theta_{k}\right],
$$

then $\operatorname{det} \Theta\left(\mathcal{D} B_{n, k}\right)=x_{1} x_{2} \cdots x_{k} \operatorname{det} \Theta\left(\mathcal{D}_{n}\right)= \pm Q\left(\mathcal{D} B_{n, k}\right)$, so we do indeed have a basis. Thus $\exp \left(\mathcal{D} B_{n, k}\right)=\{1,3,5, \ldots, 2 n-3, n-1+k\}$ as desired. The $\mathcal{D} B_{n, k}$ were first considered by Zaslavsky [57]. Bases for the module of derivations associated to other hyperplane arrangements interpolating between the three infinite Weyl families have been computed by Józefiak and me [32]. Edelman and Reiner [21] have determined all free arrangements lying between $\mathcal{A}_{n}$ and $\mathcal{B}_{n}$. It is still an open problem to find all the free subarrangements of $\mathcal{B}_{n}$ which do not contain $\mathcal{A}_{n}$.

Related to these interpolations are the notions of inductive and recursive freeness. If $\mathcal{A}$ is any hyperplane arrangement and $H \in \mathcal{A}$, then we have the corresponding deleted arrangement in $\mathbb{R}^{n-1}$

$$
\mathcal{A}^{\prime}=\mathcal{A} \backslash H
$$


and the restricted arrangement in $\mathbb{R}^{n-1}$

$$
\mathcal{A}^{\prime \prime}=\left\{H^{\prime} \cap H: H^{\prime} \in \mathcal{A}^{\prime}\right\} \text {. }
$$

In this case $\left(\mathcal{A}, \mathcal{A}^{\prime}, \mathcal{A}^{\prime \prime}\right)$ is called a triple of arrangements. Of course $\mathcal{A}^{\prime}$ and $\mathcal{A}^{\prime \prime}$ depend on $H$ even though the notation does not reflect this fact. Also if $\mathcal{A} \subseteq \mathcal{B}_{n}$ then one can mirror these two operations by defining deletion or contraction of corresponding edges in $G_{\mathcal{A}}$. The following Deletion-Restriction Theorem shows how the characteristic polynomials for these three arrangements are related.

Theorem $5.3([18],[56])$. If $\left(\mathcal{A}, \mathcal{A}^{\prime}, \mathcal{A}^{\prime \prime}\right)$ is a triple of arrangements, then

$$
\chi(\mathcal{A}, t)=\chi\left(\mathcal{A}^{\prime}, t\right)-\chi\left(\mathcal{A}^{\prime \prime}, t\right) .
$$

For freeness, we have Terao's Addition-Deletion Theorem. Note that its statement about the exponents follows immediately from the previous result.

Theorem 5.4 ( $[50])$. If $\left(\mathcal{A}, \mathcal{A}^{\prime}, \mathcal{A}^{\prime \prime}\right)$ is a triple of arrangements, then any two of the following statements implies the third:

$$
\begin{aligned}
& \mathcal{A} \text { is free with } \exp \mathcal{A}=\left\{e_{1}, \ldots, e_{n-1}, e_{n}\right\}, \\
& \mathcal{A}^{\prime} \text { is free with } \exp \mathcal{A}^{\prime}=\left\{e_{1}, \ldots, e_{n-1}, e_{n}-1\right\}, \\
& \mathcal{A}^{\prime \prime} \text { is free with } \exp \mathcal{A}^{\prime \prime}=\left\{e_{1}, \ldots, e_{n-1}\right\}
\end{aligned}
$$

Continuing to follow [50], define the class $\mathcal{I} F$ of inductively free arrangements to be those generated by the rules

(1) the empty arrangement in $\mathbb{R}^{n}$ is in $\mathcal{I} F$ for all $n \geq 0$;

(2) if there exists $H \in \mathcal{A}$ such that $\mathcal{A}^{\prime}, \mathcal{A}^{\prime \prime} \in \mathcal{I} F$ and $\exp \mathcal{A}^{\prime \prime} \subset \exp \mathcal{A}^{\prime}$, then $\mathcal{A} \in \mathcal{I} F$.

So to show that $\mathcal{A}$ is inductively free, we must start with an arrangement which is known to be inductively free and add hyperplanes one at a time so that (2) is always satisfied. If $\mathcal{F}$ denotes the class of free arrangements, then Theorem 5.4 shows that $\mathcal{I} F \subseteq \mathcal{F}$ and one can come up with examples to show that the inclusion is in fact strict. On the other hand, it is not hard to show using interpolating arrangements that $\mathcal{A}_{n}, \mathcal{B}_{n}$ and $\mathcal{D}_{n}$ are all inductively free. Ziegler [60] has introduced an even larger class of arrangements. The class of recursively free arrangements, $\mathcal{R} F$, is gotten by using the same two conditions as for $\mathcal{I} F$ plus

(3) if there exists $H \in \mathcal{A}$ such that $\mathcal{A}, \mathcal{A}^{\prime \prime} \in \mathcal{R} F$ and $\exp \mathcal{A}^{\prime \prime} \subset \exp \mathcal{A}$, then $\mathcal{A}^{\prime} \in \mathcal{I} F$.

It can be shown that $\mathcal{I} F \subset \mathcal{R} F$ strictly, but it is not known whether every free arrangement is recursively free.

\section{Supersolvability}

In this section we will look at a combinatorial method of Stanley [44] which applies to lattices in general, not just those which arise from arrangements. First, however, we must review an important result of Rota [40] which gives a combinatorial interpretation to the Möbius function of a semimodular lattice.

A lattice $L$ is modular if for all $x, y, z \in L$ with $y \leq z$ we have an associative law

$$
y \vee(x \wedge z)=(y \vee x) \wedge z \text {. }
$$

A number of natural examples, e.g., the partition lattice, are not modular but satisfy the weaker condition

$$
\text { if } x \text { and } y \text { both cover } x \wedge y \text {, then } x \vee y \text { covers both } x \text { and } y
$$




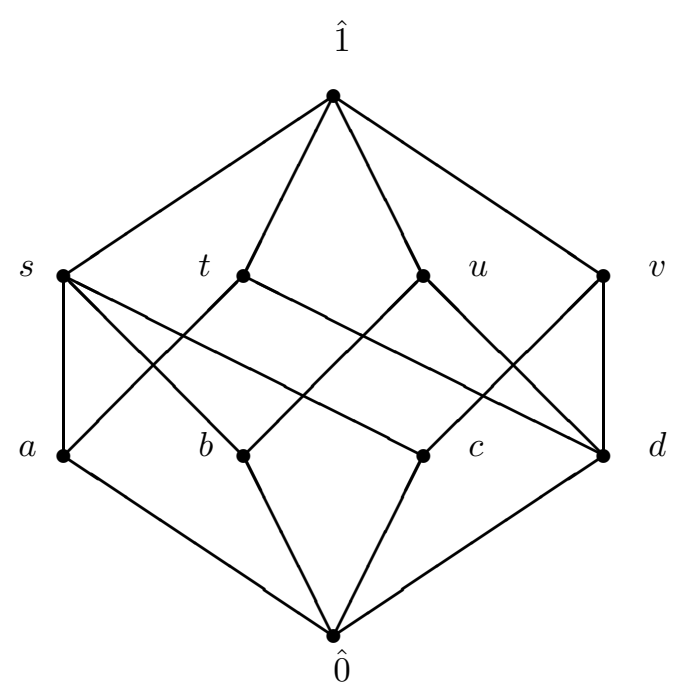

Figure 4. A lattice $L$

for all $x, y \in L$. (If $x, y \in L$ then $x$ covers $y$ if $x>y$ and there is no $z$ with $x>z>y$.) Such lattices are called semimodular. Lattice $L$ is modular if and only if both $L$ and its dual $L^{*}$ (where the order relation is reversed) are semimodular.

A set of important elements of $L$ are its atoms which are all elements $a$ covering $\hat{0}$. We let $A(L)$ denote the atom set of $L$. If $L$ is semimodular, then one can show that it is ranked. Furthermore, if $B \subseteq A(L)$ then one can prove that

$$
\rho(\bigvee B) \leq|B| .
$$

We will call $B$ independent and a base for $x=\bigvee B$ if (14) holds with equality. This terminology comes from the theory of vector spaces. Indeed if one takes $L$ to be the lattice of all subspaces of $\mathbb{F}_{q}^{n}\left(\mathbb{F}_{q}\right.$ a finite field) ordered by inclusion, then atoms have dimension 1 and lattice independence corresponds to independence of lines. A circuit is a dependent set which is minimal with respect to inclusion. If arrangement $\mathcal{A} \subseteq \mathcal{A}_{n}$ has graph $G=G_{\mathcal{A}}$, then the atoms of $L(\mathcal{A})$ are edges of $G$ and a circuit of $L(\mathcal{A})$ forms a circuit in $G$ in the usual graph-theoretic sense.

Now impose an arbitrary total order on $A(L)$ which will be denoted $\unlhd$ so as to distinguish it from the partial order $\leq$ on $L$. A circuit $C \subseteq A(L)$ gives rise to a broken circuit, $\bar{C}$, obtained by removing the minimal element of $C$ in $\unlhd$. A set $B \subseteq A(L)$ is $N B C$ (No Broken Circuit) if $B$ does not contain any of the $\bar{C}$. Note that in this case $B$ must be independent and so a base for $\bigvee B$. To illustrate, consider the semimodular lattice $L$ in Figure 4. If we order the atoms $a \triangleleft b \triangleleft c \triangleleft d$, then $L$ has unique circuit $C=\{a, b, c\}$ with associated broken circuit $\bar{C}=\{b, c\}$. Comparing the number of NBC bases of each element with its Möbius function in the following table:

\begin{tabular}{r|cccccccccc} 
element $x$ & $\hat{0}$ & $a$ & $b$ & $c$ & $d$ & $s$ & $t$ & $u$ & $v$ & $\hat{1}$ \\
\hline NBC bases of $x$ & $\emptyset$ & $a$ & $b$ & $c$ & $d$ & $a, b$ & $a, d$ & $b, d$ & $c, d$ & $a, b, d$ \\
& & & & & & $a, c$ & & & & $a, c, d$ \\
$\mu(x)$ & +1 & -1 & -1 & -1 & -1 & +2 & +1 & +1 & +1 & -2
\end{tabular}


should lead the reader to a conjecture! This is in fact the famous result of Rota referred to earlier and is usually called the NBC Theorem.

Theorem $6.1([40])$. Let $L$ be a semimodular lattice. Then for any total ordering $\unlhd$ of $A(L)$ we have

$$
\mu(x)=(-1)^{\rho(x)}(\text { number of NBC bases of } x) .
$$

In order to apply the NBC Theorem to our factorization problem, we will need to make an additional restriction on $L$. Write $x M z$ and call $x, z$ a modular pair if equation (13) is satisfied for all $y \leq z$. Furthermore $x \in L$ is a modular element if $x M z$ and $z M x$ for every $z \in L$. For example, if $L=L\left(\mathcal{A}_{n}\right)$ or $L\left(\mathcal{B}_{n}\right)$, then an element corresponding to a graph $K_{W}$ which has a complete component on the vertex set $W \subseteq\{1,2, \ldots, n\}$ (all possible edges from the parent graph, $G_{\mathcal{A}_{n}}$ or $G_{\mathcal{B}_{n}}$, between elements of $W$ ) and all other components trivial (isolated vertices) is modular. A semimodular lattice is supersolvable if it has a maximal chain of modular elements. The lattice of subgroups of a finite supersolvable group (one possessing a normal series where quotients of consecutive terms are cyclic) is supersolvable. From the previous example we see that $L\left(\mathcal{A}_{n}\right)$ and $L\left(\mathcal{B}_{n}\right)$ are supersolvable. However, it is not true that $L\left(\mathcal{D}_{n}\right)$ is supersolvable as we will see later.

Now any chain $\hat{0}=x_{0}<x_{1}<\ldots<x_{n}=\hat{1}$ in $L$ defines a partition of the atoms $A(L)$ into subsets

$$
A_{i}=\left\{a \in A(L): a \leq x_{i} \text { and } a \not \leq x_{i-1}\right\}
$$

called levels. A total order $\unlhd$ on $A(L)$ is said to be induced if it satisfies

$$
\text { if } a \in A_{i} \text { and } b \in A_{j} \text { with } i<j \text {, then } a \triangleleft b \text {. }
$$

With these definitions we can state one of Stanley's main results [44] about semimodular supersolvable lattices. It shows that their characteristic polynomials factor over $\mathbb{Z}_{\geq 0}$ because the roots are the cardinalities of the $A_{i}$.

Theorem 6.2 ([44]). Let $L$ be a semimodular supersolvable lattice, and suppose $\hat{0}=x_{0}<x_{1}<\ldots<x_{n}=\hat{1}$ is a maximal chain of modular elements of $L$. Then for any induced total order $\unlhd$ on $A(L)$

(1) the $N B C$ bases of $L$ are exactly the sets of atoms gotten by picking at most one atom from each $A_{i}$,

(2) $\chi(L, t)=\left(t-\left|A_{1}\right|\right)\left(t-\left|A_{2}\right|\right) \cdots\left(t-\left|A_{n}\right|\right)$.

Proof. The proof that (1) implies (2) is so simple and beautiful that I cannot resist giving it. The coefficient of $t^{n-k}$ on the right side of $(2)$ is $(-1)^{k}$ times the number of ways to pick $k$ atoms from exactly $k$ of the $A_{i}$. But by (1) this is up to sign the number of NBC bases of elements at rank $k$. Putting back in the sign and using the NBC Theorem, we see that this coefficient is the sum of all the Möbius values for elements of rank $k$, which agrees with the corresponding coefficient on the left side.

As an example, consider the chain of graphs with a single nontrivial complete component

$$
\hat{0}=K_{\{1\}}<K_{\{1,2\}}<\ldots<K_{\{1,2, \ldots, n\}}=\hat{1}
$$

in $\Pi_{n}=L\left(\mathcal{A}_{n}\right)$. Then $A_{k}$ is the set of all positive edges from $k+1$ to $i, i<k+1$, and so $\left|A_{k}\right|=k$. Thus $\chi\left(\Pi_{n}, t\right)=\prod_{i=1}^{n-1}(t-i)$ as before. Using the analogous chain 
in $L\left(\mathcal{B}_{n}\right)$ (which starts at $K_{\emptyset}$ ) gives $A_{k}$ as containing all edges $i k^{ \pm}, i<k$, and all half-edges $j^{h}, j \leq k$. So $\left|A_{k}\right|=2 k-1$ giving the usual roots. Now we can also see why $L\left(\mathcal{D}_{n}\right)$ is not supersolvable for $n \geq 4$. When $n \geq 4$ the second smallest root of $\chi\left(\mathcal{D}_{n}, t\right)$ is 3 . So if the lattice were supersolvable, then Theorem 6.2 would imply that some element $x \in L\left(\mathcal{D}_{n}\right)$ of rank two would have to cover at least $3+\left|A_{1}\right|=4$ atoms. It is easy to verify that there is no such element.

It is frustrating that $L\left(\mathcal{D}_{n}\right)$ is not supersolvable. To get around this problem, Bennett and I have introduced a more general concept [5]. Looking at the previous proof, the reader will note that it would still go through if every NBC base could be obtained in the following manner. First pick an atom from a set $A_{1}=\left\{a_{1}, a_{1}^{\prime}, a_{1}^{\prime \prime}, \ldots\right\}$. Then pick the second atom from one of a family of sets $A_{2}, A_{2}^{\prime}, A_{2}^{\prime \prime}, \ldots$ according to whether the first atom picked was $a_{1}, a_{1}^{\prime}, a_{1}^{\prime \prime}, \ldots$ respectively, where $\left|A_{2}\right|=\left|A_{2}^{\prime}\right|=\left|A_{2}^{\prime \prime}\right|=\ldots$, and continue similarly. This process can be modeled by an object which we call an atom decision tree or ADT, and any lattice admitting an ADT has a characteristic polynomial with roots $r_{i}$ equal to the common cardinality of all the sets of index $i$. It turns out that the lattices for all of the interpolating arrangements $\mathcal{D} B_{n, k}$ admit ADTs and this combinatorially explains their factorization. Barcelo and Goupil [4] have independently come up with a factorization of the NBC complex of $L\left(\mathcal{D}_{n}\right)$ (the simplicial complex of all NBC bases of a lattice) which is similar to the ADT one. Their paper also contains a nice result (joint with Garsia) relating the NBC sets with reduced decompositions into reflections of Weyl group elements.

Another way to generalize the previous theorem is to replace the semimodularity and supersolvability restrictions by weaker conditions. The new concepts are based on a generalization of the NBC theorem that completely eliminates semimodularity from its hypothesis. Let $\unlhd$ be any partial order on $A(L)$. It can be anything from a total order to the total incomparability order induced by the ordering on $L$. A set $D \subseteq A(L)$ is bounded below if for any $d \in D$ there is $a \in A(L)$ such that

(a) $a \triangleleft d$ and

(b) $a<\bigvee D$.

In other words $a$ bounds $d$ below in $(A(L), \unlhd)$ and also bounds $\bigvee D$ below in $(L, \leq)$. We say $B \subseteq A(L)$ is $N B B$ if it contains no bounded below set and say that $B$ is an $N B B$ base for $x=\bigvee B$. Blass and I have proved the following NBB Theorem which holds for any lattice.

Theorem 6.3 ([14]). Let $L$ be any lattice and let $\unlhd$ be any partial order on $A(L)$. Then for any $x \in L$ we have

$$
\mu(x)=\sum_{B}(-1)^{|B|}
$$

where the sum is over all NBB bases of $x$.

Note that when $L$ is semimodular and $\unlhd$ is total then the NBB and NBC bases coincide. Also in this case all NBC bases of $x$ have the same cardinality, namely $\rho(x)$, and so our theorem reduces to Rota's. However, this result has much wider applicability, giving combinatorial explanations for the Möbius functions of the noncrossing partition lattices and their type $B$ and $D$ analogs [33], [39], integer partitions under dominance order [16], [17], [24], and the shuffle posets of Greene [25]. 
Call $x \in L$ left modular if $x M z$ for all $z \in L$. So this is only half of the condition for modularity of $x$. Call $L$ itself left modular if

$L$ has a maximal chain $\hat{0}=x_{0}<x_{1}<\ldots<x_{n}=\hat{1}$ of left modular elements.

This is strictly weaker than supersolvability as can be seen by considering the 5 element nonmodular lattice [44, Proposition 2.2 and ff.].

In Stanley's theorem we cannot completely do away with semimodularity as we did in Rota's (the reason why will come shortly), but we can replace it with a weaker hypothesis which we call the level condition. In it we assume that the partial order $\unlhd$ has been induced by some maximal chain, i.e., satisfies (16) with "if" replaced by "if and only if".

$$
\text { If } \unlhd \text { is induced and } b_{0} \triangleleft b_{1} \triangleleft b_{2} \triangleleft \ldots \triangleleft b_{k} \text {, then } b_{0} \not \leq \bigvee_{i=1}^{k} b_{i}
$$

It can be shown that semimodularity implies the level condition for any induced order but not conversely. An LL lattice is one having a maximal left modular chain such that the induced partial order satisfies the level condition. So Theorem 6.2 generalizes to the following. Note that we must extend the definition of the characteristic polynomial since an LL lattice may not have a rank function and so the first of the two parts makes $\chi$ well-defined.

Theorem 6.4 ([14]). Let $L$ be an an LL lattice with $\unlhd$ the partial order on $A(L)$ induced by a left modular chain.

(1) The NBB bases of $L$ are exactly the sets of atoms obtained by picking at most one atom from each $A_{i}$ and all NBB bases of a given $x \in L$ have the same cardinality denoted $\rho(x)$.

(2) If we define $\chi(L, t)=\sum_{x \in L} \mu(x) t^{\rho(\hat{1})-\rho(x)}$ with $\rho$ as in (1), then

$$
\chi(L, t)=\left(t-\left|A_{1}\right|\right)\left(t-\left|A_{2}\right|\right) \cdots\left(t-\left|A_{n}\right|\right) .
$$

This theorem can be used on lattices where Stanley's theorem does not apply, e.g., the Tamari lattices [22], [23], [29] and certain shuffle posets [24]. Note also that we cannot drop the level condition, which replaced semimodularity, completely: If one considers the non-crossing partition lattice, then it has the same modular chain as $\Pi_{n}$. However, it does not satisfy the level condition and its characteristic polynomial does not factor over $\mathbb{Z}_{\geq 0}$.

I hope that you have enjoyed this tour through the world of the characteristic polynomial and its factorizations. Maybe you will feel inspired to try one of the open problems mentioned along the way.

\section{ACKNOWLEDGMent}

I would like to thank the referee for very helpful suggestions.

\section{REFERENCES}

[1] G. Andrews, "The Theory of Partitions," Addison-Wesley, Reading, MA, 1976. MR 58:27738

[2] C. A. Athanasiadis, Characteristic polynomials of subspace arrangements and finite fields, Adv. in Math. 122 (1966), 193-233. MR 97k:52012

[3] C. A. Athanasiadis and S. Linusson, A simple bijection for the regions of the Shi arrangement of hyperplanes, preprint.

[4] H. Barcelo and A. Goupil, Non-broken circuits of reflection groups and their factorization in $D_{n}$, Israel J. Math. 91 (1995), 285-306. MR 96g:20058

[5] C. Bennett and B. E. Sagan, A generalization of semimodular supersolvable lattices, J. Algebraic Combin. 72 (1995), 209-231. MR 96i:05180 
[6] A. Björner, The homology and shellability of matroids and geometric lattices, Chapter 7 in "Matroid Applications," N. White ed., Cambridge University Press, Cambridge, 1992, 226-283. MR 94a:52030

[7] A. Björner, Subspace arrangements, in "Proc. 1st European Congress Math. (Paris 1992)," A. Joseph and R. Rentschler eds., Progress in Math., Vol. 122, Birkhäuser, Boston, MA, (1994), 321-370. MR 96h:52012

[8] A. Björner and L. Lovász, Linear decision trees, subspace arrangements and Möbius functions, J. Amer. Math. Soc. 7 (1994), 667-706. MR 97g:52028

[9] A. Björner, L. Lovász and A. Yao, Linear decision trees: volume estimates and topological bounds, in "Proc. 24th ACM Symp. on Theory of Computing," ACM Press, New York, NY, 1992, 170-177.

[10] A. Björner and B. Sagan, Subspace arrangements of type $B_{n}$ and $D_{n}$, J. Algebraic Combin., 5 (1996), 291-314. MR 97g:52028

[11] A. Björner and M. Wachs, Nonpure shellable complexes and posets I, Trans. Amer. Math. Soc. 348 (1996), 1299-1327. MR 96i:06008

[12] A. Björner and M. Wachs, Nonpure shellable complexes and posets II, Trans. Amer. Math. Soc. 349 (1997), 3945-3975. MR 98b:06008

[13] A. Björner and V. Welker, The homology of " $k$-equal" manifolds and related partition lattices, Adv. in Math. 110 (1995), 277-313. MR 95m:52029

[14] A. Blass and B. E. Sagan, Möbius functions of lattices, Adv. in Math. 127 (1997), 94-123. MR 98c:06001

[15] A. Blass and B. E. Sagan, Characteristic and Ehrhart polynomials, J. Algebraic Combin. 7 (1998), 115-126. CMP 98:10

[16] K. Bogart, The Möbius function of the domination lattice, unpublished manuscript, 1972.

[17] T. Brylawski, The lattice of integer partitions, Discrete Math. 6 (1973), 201-219. MR 48:3752

[18] T. Brylawski, The broken circuit complex, Trans. Amer. Math. Soc. 234 (1977), 417-433.

[19] G. Chartrand and L. Lesniak, "Graphs and Digraphs," second edition, Wadsworth \& Brooks/Cole, Pacific Grove, CA, 1986. MR 87h:05001

[20] H. Crapo and G.-C. Rota, "On the Foundations of Combinatorial Theory: Combinatorial Geometries," M.I.T. Press, Cambridge, MA, 1970. MR 45:74

[21] P. H. Edelman and V. Reiner, Free hyperplane arrangements between $A_{n-1}$ and $B_{n}$, Math. Zeitschrift 215 (1994), 347-365. MR 95b:52021

[22] H. Friedman and D. Tamari, Problèmes d'associativité: Une treillis finis induite par une loi demi-associative, J. Combin. Theory 2 (1967), 215-242. MR 39:345; MR 39:344

[23] G. Grätzer, "Lattice Theory," Freeman and Co., San Francisco, CA, 1971, pp. 17-18, problems 26-36. MR 48:184

[24] C. Greene, A class of lattices with Möbius function $\pm 1,0$, European J. Combin. 9 (1988), 225-240. MR 89i:06012

[25] C. Greene, Posets of Shuffles, J. Combin. Theory Ser. A 47 (1988), 191-206. MR 89d:06003

[26] M. Haiman, Conjectures on the quotient ring of diagonal invariants. J. Alg. Combin. , 3 (1994), 17-76. MR 95a:20014

[27] P. Headley, "Reduced Expressions in Infinite Coxeter Groups," Ph.D. thesis, University of Michigan, Ann Arbor, 1994.

[28] P. Headley, On reduced words in affine Weyl groups, in "Formal Power Series and Algebraic Combinatorics, May 23-27, 1994," DIMACS, Rutgers, 1994, 225-242.

[29] S. Huang and D. Tamari, Problems of associativity: A simple proof for the lattice property of systems ordered by a semi-associative law, J. Combin. Theory Ser. A 13 (1972), 7-13. MR 46:5191

[30] J. E. Humphreys, "Reflection Groups and Coxeter Groups," Cambridge Studies in Advanced Mathematics, Cambridge University Press, Cambridge, 1990. MR 92h:20002

[31] M. Jambu and L. Paris, Combinatorics of inductively factored arrangements, European J. Combin. 16 (1995), 267-292. MR 96c:52022

[32] T. Józefiak and B. E. Sagan, Basic derivations for subarrangements of Coxeter arrangements, J. Algebraic Combin. 2 (1993), 291-320. MR 94j:52023

[33] G. Kreweras, Sur les partitions non-croisées d'un cycle, Discrete Math. 1 (1972), 333-350. MR 46:8852

[34] S. Linusson, Möbius functions and characteristic polynomials for subspace arrangements embedded in $B_{n}$, preprint. 
[35] S. Linusson, Partitions with restricted block sizes, Möbius functions and the $k$-of-each problem, SIAM J. Discrete Math. 10 (1997), 18-29. MR 97i:68095

[36] P. Orlik and H. Terao, "Arrangements of Hyperplanes," Grundlehren 300, Springer-Verlag, New York, NY, 1992. MR 94e:52014

[37] A. Postnikov, "Enumeration in algebra and geometry," Ph.D. thesis, M.I.T., Cambridge, 1997.

[38] A. Postnikov and R. P. Stanley, Deformations of Coxeter hyperplane arrangements, preprint.

[39] V. Reiner, Non-crossing partitions for classical reflection groups, Discrete Math. 177 (1997), 195-222. CMP 98:05

[40] G.-C. Rota, On the foundations of combinatorial theory I. Theory of Möbius functions, $Z$. Wahrscheinlichkeitstheorie 2 (1964), 340-368. MR 30:4688

[41] K. Saito, Theory of logarithmic differential forms and logarithmic vector fields, J. Fac. Sci. Univ. Tokyo Sec. 1A Math. 27 (1980), 265-291. MR 83h:32023

[42] J. Y. Shi, The Kazhdan-Lusztig cells in certain affine Weyl groups, Lecture Notes in Math., Vol. 1179, Springer-Verlag, New York, NY, 1986. MR 87i:20074

[43] J. Y. Shi, Sign types corresponding to an affine Weyl group, J. London Math. Soc. 35 (1987), 56-74. MR 88g:20103b

[44] R. P. Stanley, Supersolvable lattices, Algebra Universalis 2 (1972), 197-217. MR 46:8920

[45] R. P. Stanley, "Enumerative Combinatorics, Volume 1," Cambridge University Press, Cambridge, 1997. MR 98a:05001

[46] R. P. Stanley, Hyperplane arrangements, interval orders, and trees, Proc. Nat. Acad. Sci. 93 (1996) 2620-2625. MR 97i:52013

[47] S. Sundaram, Applications of the Hopf trace formula to computing homology representations, Contemp. Math. 178 (1994), 277-309. MR 96f:05193

[48] S. Sundaram and M. Wachs, The homology representations of the $k$-equal partition lattice, Trans. Amer. Math. Soc. 349 (1997) 935-954. MR 97j:05063

[49] S. Sundaram and V. Welker, Group actions on arrangements of linear subspaces and applications to configuration spaces, Trans. Amer. Math. Soc. 349 (1997) 1389-1420. MR 97h:52012

[50] H. Terao, Arrangements of hyperplanes and their freeness I, II, J. Fac. Sci. Univ. Tokyo, 27 (1980), 293-320. MR 84i:32016a; MR 84i:32016b

[51] H. Terao, Generalized exponents of a free arrangement of hyperplanes and the ShepherdTodd-Brieskorn formula, Invent. Math. 63 (1981), 159-179. MR 82e:32018b

[52] H. Terao, Free arrangements of hyperplanes over an arbitrary field, Proc. Japan Acad. Ser. A Math 59 (1983), 301-303. MR 85f:32017

[53] H. Terao, The Jacobians and the discriminants of finite reflection groups, Tôhoku Math. J. 41 (1989), 237-247. MR 90m:32028

[54] H. Terao, Factorizations of Orlik-Solomon algebras, Adv. in Math. 91 (1992), 45-53. MR 90m:32028

[55] H. S. Wilf, "Generatingfunctionology," Academic Press, Boston, MA, 1990. MR 95a:05002

[56] T. Zaslavsky, "Facing up to arrangements: Face-count formulas for partitions of space by hyperplanes," Memoirs Amer. Math. Soc., No. 154, Amer. Math. Soc., Providence, RI, 1975. MR 50:9603

[57] T. Zaslavsky, The geometry of root systems and signed graphs, Amer. Math. Monthly 88 (1981), 88-105. MR 82g:05012

[58] T. Zaslavsky, Signed graph coloring, Discrete Math. 39 (1982) 215-228. MR 84h:05050a

[59] T. Zaslavsky, Chromatic invariants of signed graphs, Discrete Math. 42 (1982) 287-312. MR 84h:05050b

[60] G. Ziegler, Algebraic combinatorics of hyperplane arrangements, Ph. D. thesis, M.I.T., Cambridge, MA, 1987.

Department of Mathematics, Michigan State University, East Lansing, Mi 48824-1027

E-mail address: sagan@math.msu.edu 